\title{
Overcoming University Students' Alternative Conceptions in Newtonian Mechanics
}

\author{
Ahcene Serhane ${ }^{1,}$, , Mahdi Debieche ${ }^{1}$, Boudhar Karima ${ }^{1}$, Abdelhamid Zeghdaoui ${ }^{2}$ \\ ${ }^{1}$ Physics Department of Ecole Normale Supérieure, Vieux-kouba, Algiers, Algeria \\ ${ }^{2}$ Chemistry Departement of Ecole Normale Supérieure, Vieux-kouba, Algiers, Algeria
}

Email address:

acheneserhane@yahoo.com (A. Serhane)

*Corresponding author

\section{To cite this article:}

Ahcene Serhane, Mahdi Debieche, Boudhar Karima, Abdelhamid Zeghdaoui. Overcoming University Students' Alternative Conceptions in Newtonian Mechanics. American Journal of Networks and Communications. Vol. 9, No. 2, 2020, pp. 22-29.

doi: 10.11648/j.ajnc.20200902.12

Received: July 28, 2020; Accepted: October 19, 2020; Published: December 22, 2020

\begin{abstract}
Students' alternative frameworks and prior conceptions about interactions forces «traditionally, known as Newton's third law» have been largely investigated. The various investigations clearly show that students very often fail to apply Newton's laws of motion in general to everyday situations and third law in particular. This study highlights some of the serious difficulties students undergo with reciprocal interactions and circular motions, taking advantage of previous studies and/or surveys we managed to reveal and identify a wide range of misconceptions among students, enabling teachers to set appropriate strategies to overcome them. Closely related to students' misconceptions in Newton's law of motion, there are still serious difficulties facing students to differentiate between real and fictitious forces, especially when tackling dynamics problems involving circular motion. An approach to teaching mutual interactions and the meaning of centripetal force is suggested that focuses on reconsidering and/or refining students' intuitive thinking on the nature of mutual interactions. However, the essence of this argument is that the teaching of science is not a process by which the wrong ideas are substituted by the correct ones; but students should know where they went wrong and why.
\end{abstract}

Keywords: Overcoming, Difficulties, Misconceptions, Interactions, Students, Centripetal Force

\section{Introduction}

Physics has been often recognized by a great majority of students as being one of the most difficult subjects in pure science. The reasons and/or arguments as provided by students for this are multiple and diverse, including for example, "... I have to think too deeply" or "... I need to have a good memory", or simply some of the law does not make sense at all"! "Whatever the specific argument and/or reason presented by students, the majority of teachers and / or science educators would certainly agree that conceptual difficulties encountered by students can be severe at times, so severe that many students are unable to place that concept in perspective" Hugh G. Jones and Robert J. Mooney [1]. The result is that students' knowledge and physics understanding is frequently fragmented and consequently they never come perceive a unity of the subject.

\section{Literature Review}

Antwi et al [2] found that most students understand some concepts in mechanics such as circular motion differently. He proved that being able to solve difficult mathematical problem sets is not an assurance for a complete conceptual understanding. Similarly, the work of Duman, Demerci, and Sekercioglu [3] and Vyas [4] were specific in finding out misconceptions and alternative conceptions on circular motion. In these studies, it was revealed that students in the university have difficulty in mastering, applying and interpreting fundamental concepts in mechanics such as circular motion concepts. Likewise, Viridi, Moghrabi and Nasri [5] made the same findings, but did a more practical work in probing students understanding of circular motion using ordinary appliances, train toy, digital camera and android-based smartphone. In addition, another group of 
studies that are related to circular motion were on designing and implementing teaching and learning strategies that could increase the knowledge and address alternative conceptions in circular motion. Seattha, Yuenyong and Art-in [6] developed a very practical approach in teaching circular motion by using science, technology and society (STS) approach in teaching circular motion to understand the context of science, technology, engineering and mathematics (STEM). Similarly, Stinner [7] advocated in using more common and natural phenomena rather than focusing on textbooks in his strategy called 'linking the book of nature and the book of science' to understand circular motion. Meanwhile, the use of computer and Internet technology [8, 9] and inquiry-based strategy [10] were also found to be effective and efficient in teaching circular motion. Although, a number of studies have argued that it is nearly impossible to change preconceptions of students on physics concepts [11-13]. According to Ozdemir [11], misconceptions either would tend to coexist with the scientific conceptions or will reappear after sometime even after giving a successful teaching [12, 13]. Finally, another group of studies were on developing tools for assessment of conceptual understanding on circular motion. Among these assessment tools were the three-tier misconception test [14] and the use of diagram [15] in probing conceptual understanding of circular motion concepts. This study was focused on assessing alternative conceptions of university students on circular motion specifically on linear speed and rotational speed, velocity, centripetal acceleration and centripetal force. It combined a number of assessment tools including analysis of diagrams, practical phenomena, written test, individual and group interviews. It also focused on finding out inconsistencies in the understanding of students on the above-mentioned concepts. Circular movement is one of the most important topics that resonates frequently in physics courses. Besides that, it hides some of the most difficult concepts to overcome or grasp by students, despite the formal education they received. Studies have shown that many students including those at the tertiary level encountered difficulties in understanding the concepts of circular motion. Finley, Stewart and Yarroch (1982) [16] found that circular motion was the most difficult concept for students. Salvage and William (1989) [17] credited some of the students' confusions to the introduction of centrifugal force.

Warren (1971) [18] had asked 148 English university entrants in engineering and science to draw an arrow showing the resultant force acting on a car travelling at constant speed along a horizontal, circular road. About $40 \%$ of the students indicated the resultant force in the direction of the motion, $26 \%$ of the students showed a radial and inward force while $14 \%$ showed a radial and outward resultant force. Viennot (1979) [19] used paper and pencil tests to investigate spontaneous reasoning in elementary dynamics to physics students in France, Belgium and Britain. She argued that many university students treated circular motion as an example of an equilibrium situation, and this led them to invent an outward centrifugal force to counterbalance the inward centripetal force. She also proposed an explanation of students' spontaneous reasoning in terms of an assumed linear relationship between force and velocity: Zero velocity implied zero force. Thus, if an object has no radial velocity, then it has no radial force acting on it. In the United States of America, McCloskey, Caramazza and Green (1980) [20] asked 50 undergraduate students at John Hopkins University to sketch the subsequent paths of a moving ball emerging from a simple C-shaped tube, a double $\mathrm{C}$-shaped tubes and a spiral tube. The students were also asked to describe the path of a pendulum bob cut from its string at a certain point of circular motion. A total of $36 \%$ of the paths drawn by the students were of curvilinear paths. More specifically, for the spiral tube problem, $51 \%$ of the students thought that the ball would follow a curved path after emerging from the tube. For the simple $\mathrm{C}$-shaped and double C-shaped tubes, the curved paths drawn constituted respectively $33 \%$ and $30 \%$ of the sample. Similarly, about $30 \%$ of the students believed that the ball would continue to follow a curved path after the string broke. McClockey et al. [21] stated that the above students' beliefs were reminiscent of the medieval theory of impetus. In Australia, Gunstone [22] and Gardner respectively investigated the students' pre- and postinstructional alternative frameworks in circular motion using the same paper and pencil test. Among the questions in the test were: a number of questions concerned with drawings of objects in circular motion; questions which asked for labeled arrows to indicate the forces acting on objects; questions which asked the total force acting on objects. Gardner (198 [23] identified six alternative conceptual frameworks that the students used to account for the dynamics of circular motion. Among the frameworks identified were: (a) the Motive Force Framework, (b) the Equilibrium Framework Type I Absence of Radial Forces whereby the students treated circular motion at constant speed as rectilinear motion at constant speed, (c) the Equilibrium Framework Type II Two Counter-Balancing Radial Forces in which the students' explanations postulated a balance between a centripetal force and an equal and opposite centrifugal force. Gunstone (1984) reported that a total of $84 \%$ of the students indicated the Motive-Force Framework while $12 \%$ of the students showed a form of centrifugal force concept. Similarly, Searle (1985) [24] administered a test that contained seven questions of the DOE (Demonstrate, Observe and Explain) type, to 19 firstyear engineering students. The test was designed to investigate the following misconceptions in classical mechanics: (i) motion implied force belief, (ii) the impetus theory, and (iii) the commonly held view that an outward centrifugal force acted on objects moving in a circle. Among the six misconceptions held by the students included the impetus theory, motion or displacement implied force and equilibrium framework. In Jamaica, Whiteley (1995) [25] asked 115 upper six students of advanced level physics to give a 'reasonable one-word answer' to the question: "What hold up the Moon?" He found out that some of the 58 students who had answered in the 'gravity' or 'centripetal force' categories indicated that they actually believed in two 
forces (gravity and centripetal force) rather than recognizing that gravity was the centripetal force that held up the Moon. A small number (6) of students had the belief that a balancing force was needed to counter the attraction of the Earth for the Moon by identifying the attraction of other planets such as Mars or an outward centrifugal force in order to balance the gravity. The study reported in this paper builds on the studies mentioned above and sought to investigate the students' understanding of the basic concepts in circular motion.

\subsection{Concepts in Teaching and Learning}

Physics is introduced to upper secondary students (16 years old). However, it is a general fact that most students have a negative perspective about Physics. This is because they do not obtain high marks in examination even though they have tried their best. This phenomenon is caused by misconception and lack of understanding about basic Physics concepts. Many researchers have shown that students tend to develop the understanding about natural phenomenon before formal teaching was conducted. Ideas developed by students which differ from scientific explanation are known as misconceptions [26] and these misconceptions are too strong to overcome and can inhibit the process of teaching and learning (Gunstone, 1995) [27] Initial ideas held by students are very difficult to change by teacher despite being presented with scientific concepts (Tsai, 1999; Sencar \& Eryilmaz, 2004) [28, 29]. Teachers should identify students' misconception before conducting formal teaching so that their misconception can be changed to scientific concept after the formal teaching and learning process (Wong \& Seth, 2008) [30]. Halim et al. (2009) [31]. state that to build an effective model of teaching and learning for teaching physics it should begin by exploring or identifying difficulties and misunderstanding faced by students using diagnostic tests. Several studies have found that misconception still exist even when the students have attended more Physics class. The same misconception occurs and exists at primary and up to university level. This shows that normal or regular teaching and learning process is unable to overcome misconception if the focus of the teaching is not targeted on the misconception. One of the difficulties to overcome the misconception is that the teachers themselves are not being trained to overcome the misconception. Osman et al. (2006) [32]. added, even though the role of quality Science teacher has been realized, but like many countries (e.g. Pakistan, Australia, New Zealand, United States of America and Britain), Malaysia is facing the problem of inadequate trained Science teachers especially in the teaching of Physics, Chemistry and Mathematics. As a result, teachers who are not trained in teaching Science subjects are often required to teach Science. If nothing is done to help this group of teachers, they probably are unable to implement Science curriculum effectively. The study of student misconceptions in Physics has become both an important concern for Physics education research and a valuable tool for the practicing physics teacher (Styer, 1995;
Abell \& Lederman, 2007) [33, 34]. Although some misconceptions are difficult to overcome, teachers must be aware about the existence of the misconception and keep addressing to overcome students' misconceptions so that students will have conceptual understanding. The study of this phenomenon needs to be solved by taking specific action namely by having collaborative relationship between university professors and student teachers to plan a specific action to be implemented. This has encouraged the researchers to explore physics misconception in the topic of mechanics and the use of forces.

\subsection{The Origin and Background of Misconceptions}

"Many researchers agree that most of these conceptions and/or alternative conceptions originate from the implementation of simplified thinking (reasoning) schemes" Redish E F (2004) [35] but some of them seem to be additionally reinforced by students' everyday experience and/or observation. "Such ideas are found more frequently in classical mechanics, the area of physics closest to students' experience" Planinic M, boone W J, Krsnik R and Beilfuss M $\mathrm{L}$ (2006) [36]. It is the association of reasoning and everyday experience that makes these ideas reinforced as firm alternative conceptions that are very highly resistant to change (It is well known that Newtonian mechanics appears both essential to know as the historical and pedagogical foundation of physical theory and a considerable difficulty.

\subsection{Newtonian Mechanics and Students Conceptions}

"Mechanics is typically the area of opposition and/or confrontation between ideas and reasoning of common sense and the theory taught." Viennot. L (2002) [37]. It is not that it is difficult to report or retain the fundamental law of dynamics for a particle $\vec{F}=m \vec{a}$ in usual notation, the law of interactions, or the third law of Newton, for two particles $\vec{F}_{1 / 2}=-\vec{F}_{2 / 1}$ and their corollaries for sets of particles or systems namely the theorem of the center of inertia $\left(\mathrm{F}_{\mathrm{ext}}=\right.$ $\mathrm{ma}_{\mathrm{G}}$ ) and the law of interactions For two objects (A and B): $\vec{F}_{A / B}=-\vec{F}_{B / A}$ always in usual notation". Simply, for many situations in mechanics, the answers dictated by the common sense and those which it allows to accept without hesitation are in total opposition to the Newtonian analysis. Thus, each time the speed of a mobile and the resultant of the force applied to this it is clearly not simultaneously zero or of the same direction, it seemed necessary to imagine what is necessary as force in the direction intended for that the two vectors in question slit on each other. There is no doubt that Newton's third law is difficult to comprehend, and is even known to harbor some of the last misconceptions to be overcome in the transition to Newtonian thinking Hestenes, D., Wells, M. and Swachamer, G. (1992) [38]. Moreover, "people often admit to having problems in believing Newton's third law to be true in all the circumstances which it is applied" Gauld, C. (1998) [39]. The third law is fundamental and essentially defines what counts as a force: a force always involves an interaction between two objects. 
Brown (1989) explains that understanding Newton's third law requires one to understand that forces arise from interaction. In this context, the third law actually underpins the first and second laws,

and many of the common misconception relating to the first two laws can be attributed to the failure to grasp the significance of the third law, or more specifically that: if body X exerts a force on body Y, body Y simultaneously exerts on body $\mathrm{X}$ a force equal in magnitude and opposite in direction. However, in our everyday life, we usually experience asymmetric situations. If, say, one person pushes another, usually, the stronger one wins. Interpreting the term interaction by a "conflict metaphor" [38]. i.e. as a struggle between opposing forces, may lead one to apply in such situations a kind of dominance principle and think that the "more forceful" exerts the greater force. Here "more forceful" can mean "bigger", greater mass", or "more active" [38]. Every day experience, for instance, make it counterintuitive that a large, rapidly moving object and a small, slowly moving object should exert the same force on each other when they collide. Instead it much more intuitive (but wrong) to relates the forces in the collision to the 'forces' in the moving objects and to believe that large, rapidly moving objects have or (embody) large internal forces and exert large forces on other objects while the forces exerted by small, slowly moving objects are correspondingly smaller. Newton's 3rd law as manifested in static situations is also hard for students to grasp. According to Montanero et al. (2002) [40], one implicit theory used to explain the behavior of two bodies with one is resting on top of the other is that the lower body possesses a passive force of resistance, either as a simple impediment to penetration or as an intrinsic property of its mass (or weight). The nature of this resistance is such that, at times, it cannot be regarded as a force.

\subsection{Overcoming Newton's Third Law Misconception}

Therefore, when attempting to induce conceptual change (a transition from intuitive, not accepted scientifically to scientific accepted ideas) in students, the teacher has to be prepared to challenge both the reasoning and experience aspects of resistant alternative conceptions. It is not enough experimental evidence that a personal idea about some phenomenon might be wrong and offer the new idea instead. Unless the reasoning way to the new idea is also provided, no conceptual change will take place. I have often noticed that students have troubles and doubts accepting a new idea until they realize where their reasoning went wrong. A good example of this is Newton's third law. Students' difficulties with this law in mechanics are well explored and documented by many researchers (David Maloney, David E Brown, Clement J, Viennot, L. Terry \& jones and Warren, J W). In fact, not only students are concerned with Newton's third law difficulties, teachers and even professional physicists face some troubles as well, as it was noticed by C. Hellingman: [41] "We face the undeniable fact, hard as it is to believe, that not only students but also professional physicists to quite a large extent do not have a full understanding of force concept. It would be worthwhile to do research among professional physicists instead of among students." There is also evidence that these difficulties extend beyond mechanics and influence other area in physics. Newton's third law causes few difficulties in symmetrical situations (the interacting objects have equal masses), but in very asymmetrical situations student often claim that the two interacting forces are not equal (the massive body exerts a stronger force). It is not that students do not understand Newton's third law or do not remember its mathematical formulation $\vec{F}_{A / B}=-\vec{F}_{B / A}$ in usual notation, they simply do not trust it one of my students stood up in the classroom and openly said: " I know that forces should be equal between the horse and the cart, we have been told that many times, but that does not make any sense." Students start from the obvious fact that action and reaction have different impacts in terms of either acceleration or deformation of interacting bodies, simple analysis of the problem leads to the conclusion that the forces producing the largest impacts are the greatest. I think even when the teacher can present experimental evidence that the forces are equal, in most cases that alone will not lead to conceptual change because most of students cannot reconcile this experimental out-come with their reasoning. However, if the teacher is aware of the underlying reasoning problem, he or she can prompt students to first predict the effects of equal forces on bodies of different masses using Newton's 2nd law. Students will oftenwithout further thinking-follow their simplified line analysis (reasoning or thinking) in the first step, they will predict that equal forces produce equal effect, but in the next step, they will realize their mistake. If the teacher gives them enough time to conclude by themselves that equal forces really can produce different effect (it may take some time even for good students), and to realize that the effect also depend on the mass of the body, the great majority of students will accept Newton's third law. The benefit of such intervention is not only the acceptance of Newton's third law, but more crucial, a significant correction of their alternative framework. It is also very important to emphasize the verbalization of ideas as a crucial part of the development of students reasoning, student should not have lack of using abstract physics terms such as "force", and instead, using everyday language (push, pull, etc..), such as these should not be taken lightly, Vygotsky, for example, saw language as the principal mediator of all high mental functions (see Vygotsky 1934/1986) and therefore, as a sine qua non of mental growth. It is more natural to describe the real world using physics terminology. Being aware that language plays a crucial role in the process of conceptual growth, it is so important that teachers use and encourage students to use force and other physical terms when explaining real situations involving Newton's third law. This will make the explanation more accurate and therefore might lead to a better understanding of the law and its applicability in real situations. In this context, it is worth noting that textbooks, regrettably in my opinion, often continue to use the old formulation of the 3rd law in terms of action/reaction instead of force. Warren, J W. (1979) maintains that the third law is misunderstood because textbook and teachers present it in a form something like "action and reaction are equal and opposite". He suggests 
that the terms "action" and "reaction" imply a timesequenced cause and effect relationship whereas the forces of third law pair arise simultaneously from the same interaction. It is also likely that the use of the word "opposite" suggests the two forces must act on the same object and this perhaps why many pupils associate the third law with the condition for equilibrium. So to present the third law in an intelligible way, we should avoid the use of the words "action", "reaction" and "opposite" and instead provide a fuller and meaningful formulation of the law. The correct and/precise verbalization of ideas might also reveal the underlying reasoning, with its possible flaws, and make it easier to notice problems and intervene. Through careful listening, paying more attention to students' verbalization, the teacher can discover many interesting and important things about students reasoning in physics and sometimes even detect important alternatives conceptions. For example, when talking about the diffusion of ether vapor in the porous containing air, one student said that when ether molecules enter the vessel, molecules of air are "squeezed out". Anyone familiar with the most common alternative conceptions regarding the particulate nature of matter will recognize that the student is reasoning in terms of continuous model of matter, although he is talking about molecules at the same time. It is not at all uncommon that students superpose the particulate model on the continuous matter, the latter being much closer to their intuition. Further discussion with the student revealed that he imagined air molecules inside the vessel to be tightly packed, with no space between them. Therefore, if an ether molecule was to enter it had to "squeeze out" a molecule of air from the vessel. This important alternative conception could have easily gone unnoticed, had the expression "squeezed out" been ignored rapidly replaced by the teacher with a more adequate word.

\section{Centripetal Force Misunderstanding}

Closely related to the context of students' misconceptions in physics, is that the fact a great majority of students still hold the conception that centripetal force is a real radial force that contributes to the net radial force required to keep an object moving in a circular trajectory. This misconception seems to be rather common and/or highly resistant (propagated even among few teachers); it has been reported by other researchers: De Jong ML (1988) [42]. "What name should be used for the force required to move a mass in a circle" and Smith PA (1992) [43] "Let's get rid of centripetal force." My initial approach to overcome students' misconceptions was to emphasize in my lecture on circular motion, that centripetal force is merely the physical term given to the net radial force $\sum F r$ acting towards the center of the motion, not a real force Fr that contributes to the sum of real forces (see e.g.) Galili, I., \& Hazan, A. (2000) [44]. However, I realised that this approach, even supported by examples, was invariably not effective, a significant number of students still continue to consider centripetal force as an individual radial force (real force) when I asked them to name the individual radial forces that give rise to the net (resultant) radial force in a given situation. Today when I am dealing with circular motion, I do not tell the students that the net radial force is called centripetal force. Moreover, I do not refer to the net radial force by any other name or term because I am sure that they will have the same misconception of the new name as they do with centripetal force. I simply refer to the net radial force as the net radial force, just like for linear motion where we simply refer to the net force in a Cartesian direction as the net force in that direction. Moreover, since students know about centripetal force from their high school and pre-university physics, I tell them to forget about the term "centripetal force" in other words this term, will be banned and prohibited in my class. That it absolutely cannot be used either in conversation or in writing. This approach has been very successful. Since then, none of the students gives centripetal force as one of the individual radial forces acting on a body undergoing circular motion. The success of this approach shows that we would not have this misconception of centripetal force to deal with in the first place if the term "centripetal force" for the net radial force had never been introduced in the textbooks. It is important to note that the literature on misconceptions focuses mainly on students and not on teachers. Yip, D. Y. (1998) [45] "explains that this tendency rests on the unfounded assumption that being graduates of higher studies possess the knowledge needed for teaching the required content in the classroom". The author further states, "One source of students' misconception is the erroneous concepts propagated by the teachers themselves". Indeed, researchers have found that teachers evidence misconception as well (e.g. in physics: Galili and Hazan 2000, in physical chemistry: Gopal et al. 2004 [46]; in biology: Yip, D. Y. [45] "Therefore, it is important to consider students as well as trainees and teachers when investigating misconceptions. Textbooks also can be another cause of misconceptions.". "The third law then diverted under the guise of second law, in fact it simply violated in many affirmations, including schoolbooks. This risk is particularly relevant for contact interactions." Viennot. $\mathrm{L}$ [37] stated that the third law is then diverted under the guise of the second law, in fact, it is simply violated in affirmations, including in textbooks. This risk is particularly evident for contact interactions. Unfortunately, the term of "centripetal force" is still commonly used in textbooks today in spite of De Jong's ML [42] and Smith's PA [43] proposals against its use more than two decades ago. I hope that textbook authors and educators will concern themselves with this issue seriously.

\subsection{Implications}

This study has included consulting and reviewing an important package of previous research and field investigations related to students' physical concepts in general and circular motion in particular despite receiving formal instructions from their teachers. This finding might suggest that the students could not understand or assimilate their teachers' instruction or that the preconceptions of the students were quite resistant to change even after formal 
instruction. Hence identifying students' preconceptions is essential for the teachers to devise appropriate instructional strategies to bring about the desired conceptual change. There should be more opportunities for practical activities and discussions so that the students could clarify their views. This will help them to resolve any preconceptions that are not consistent with their observations of the ideas or phenomena discussed in the classroom. Some of the students' conceptions in circular motion were indeed the wrong conceptions such as the Aristotelian motive force and the Impetus theory of motion which were also held by the scientists in the past ages. Thus the topic of history of science should be included in the teacher-training program for physics teachers so that the trainees will have the knowledge of how the past scientists altered their wrong reasoning with the passage of time. The trainees could also compare and contrast the students' and historical misconceptions with the correct scientific conceptions. The teachers could also use the wrong reasoning of the past in convincing their students to discard any misconceptions and to acquire the accepted scientific conceptions. The findings of this study indicated that some students had a poor understanding of Newton's First and Second Laws of motion. Newton's First Law implies that if a body changes its state of motion, there is a force acting on it. Newton's Second Law states that if a body experiences an acceleration, there is a force acting on it, or conversely, if there is a force acting on a body, it will accelerate. Findings in this study showed that some students could not apply Newton's First and Second Laws in the cases of motion provided. They did not have a profound understanding of the fundamental concepts of acceleration, velocity, and force. These students did not grasp the fact that acceleration could be either due to any change of speed or any change of direction of motion. Thus, with any change of speed or change of direction of speed, a body will definitely experience a force acting on it. Teachers should provide sufficient concrete examples of different situations of motion for the students to engage in peer-group or teacher-student discussions so that the students could firmly grasp the essence of the two laws of motion. This is important, as understanding of these two laws will affect mastery of other concepts related to mechanics.

\subsection{Recommendations}

In circular motion instruction, it is important for teachers to first identify the physical nature of the force acting on an object, rather than just stating the term 'centripetal force' that enables the object to travel in circular motion. Teaching circular motion in a well-conceptualized qualitative approach without using numbers in the early part of a lesson will prevent unnecessary rote learning of formulae. This will encourage the students to understand the concepts presented. Concepts on circular motion is part of the learning competencies in the secondary school science curriculum and physical sciences in the general education curriculum. The most previous studies we have reviewed revealed a limited understanding and a considerable number of alternative conceptions on circular motion. Hence, it may be necessary to conduct an in-depth study and investigation on the reasons for failing to develop concrete conceptual mastery of circular motion concepts across level. Likewise, as these issues were proven to exist, appropriate strategies to address them may be developed and implemented. A number of literatures suggested that using inquiry-based approaches in teaching might be effective in addressing alternative conceptions and increasing students' understanding [47-49]. In addition, there may be a need to review the learning competencies related to circular motion in both the secondary science curriculum and the physical sciences of the general education curriculum. This is to ensure the appropriate, smooth and successful spiral progression in the teaching and learning of circular motion concepts across different levels. Moreover, literatures suggest that teachers' mastery of the subject matter is an important factor that affects students' achievement [50, 51]. It may be necessary to find out the conceptual mastery of the teachers on circular motion to develop issue-specific solutions related to the teaching of circular motion in the basic education and higher education. Similarly, along the conduct of this study, a similar study may be conducted across different levels, groups and races to compare and verify the results reported in this study. Moreover, an indepth study may be conducted to trace the origin of their understanding of circular motion concepts and the changes it could undergo when implementing research based pedagogies. Likewise, other phenomena with the presence of circular motion may be added to ensure consistency in the understanding of circular motion concepts such that it can come in different forms.

\section{Discussion and Conclusion}

Despite formal education, even in the most advanced countries, there are still students who are unable to describe and identify forces involved in a physical situation. An explanation for this is that, as shown in earlier studies, misconceptions are difficult to overcome (the heart of the problem is not changing wrong ideas by correct ones but students should know where they went wrong and why?). Another reason is also that students' thinking might not have reached the level of "formal thinking" in understanding the scientific concept which is often abstract. This shows that students' thinking level is very important in understanding the abstract concepts of physics such as the concept of force. According to Piaget's theory of cognitive development, students' thinking level between the ages of 11 to 15 years should have reached the level of "formal thinking", but the development is not the same for all. Therefore, the focus of intervention activities namely in improving students' thinking level should be conducted on students' misconceptions. Each student has a different thinking level therefore researcher and teacher must investigate their thinking level whether the students' thinking level has reached "formal thinking" or still in "concrete thinking" before planning any intervention strategy. By knowing the students' thinking level, teachers 
are able to design the intervention strategies to assist the student in enhancing their thinking level from "concrete thinking" to "formal thinking" which then allows the students to understand the abstract concept of forces. A module can be used to improve students' thinking level which is "Cognitive Acceleration through Science Education (CASE)". CASE is a module used to improve students' "formal thinking" in learning science. Thus, this intervention should be carried out first, before the intervention related to the teaching on the concept of forces is implemented. argues that active learning is “... techniques where students do more than simply listen to a lecture. Students are doing something including discovering, processing and applying information". Thus, by using active learning, teachers can challenge students' thinking and improve their understanding in the concept of forces.

\section{Acknowledgements}

This piece of work was supported completely by the physics department and the laboratory of $N$-body and structure of matter P. B $N^{\circ} 92$ Vieux-Kouba 16050, Algiers, ALGERIA.

ENS $=$ EcoleNormaleSupérieure, is a kind of university to train and / or prepare teachers for middle and secondary education.

Note: I would like to point out that the additive additions to the article were intended to enrich the article and benefit readers only. As for the change in the names of the authors, it was with the approval of all members of the research team, and there is no dispute or conflict between the authors at all.

\section{References}

[1] Hugh, G. Jones, and Robert, J. Mooney (1981). Phy Educ. "An approach to conceptual difficulties in physics", 16. UK.

[2] Antwi, V. et al. Students' Understanding of Some Concepts in Introductory Mechanics Course: A Study in the First Year University Students, International Journal of Educational Planning and Administration, Vol. 1, No. 1, 55-80, 2011.

[3] Duman, I., Demerci, N., and Sekercioglu, A. University Students' Difficulties and Misconceptions on Rolling, Rotational Motion and Torque Concepts. International Journal on New Trends in Education and Their Implications, Vol. 6, No. 1, 46-54, 2015.

[4] Vyas, P. Miconception in Circular Motion, International of Scientific and Engineering Research, Vol. 3, No. 12, 1-4, 2012.

[5] Viridi, S., Moghrabi, T. and Nasri, M. An Observation of a Circular Motion Using Ordinary Appliances: Train Toy, Digital Camera, and Android based Smartphone, Prosiding of Simposium Nasional Inovasi dan Pembelajaran Sains, Vol. 34, 1-7, 2013.

[6] Seattha, P., Yuenyong, C., and Art-in, S. Developing STS Circular Motion Unit for Providing Students' Perception of the Relationship between Science, Technology, Engineering and Mathematics, Mediterranean Journal of Social Sciences,
Vol. 6, No. 3, 268-275, 2015.

[7] Stinner, A. Linking 'The Book of Nature' and 'The Book of Science': Using Circular Motion as an Exemplar beyond the Textbook, Science and Education, Vol. 10, 323-344, 2001.

[8] Zhou, S. et al Inquiry style interactive virtual experiments: a case on circular motion, European Journal of Physics, Vol. 32, No. 6, 1597, 2011.

[9] Aravind, V. R. and Heard, J. W. Physics by Simulation: Teaching Circular Motion Using Applets, Latin American Journal of Physics Education, Vol. 4, No. 1, 35-39, 2010.

[10] McLaughlin, S. Rounding Up Students' Conceptions on Circular Motion, Iowa Science Teachers Journal, Vol. 33, No. 2, 7-15, 2006.

[11] Ozdemir, O. F. The Coexistence of Alternative and Scientific Conceptions in Physics, Doctoral Dissertation, The Ohio State University, 2004, online available from http://etd.ohiolink.edu/!etdsend_file?accession=osu108603335 $8 \&$ disposition $=$ inline

[12] Kim, E. and Pak, S. J. Students do not overcome conceptual difficulties after solving 1000 traditional problems, American Journal of Physics, Vol. 70, No. 7, 759- 765, 2002.

[13] Kizilcik, H. S., Ondu Celikkanli, N and Gunes, B. Change of Physics Teacher Candidates' Misconception on Regular Circular Motion by Time, Necatibey Faculty of Education Electronic Journal of Science and Mathematics Education, Vol. 9, No. 1, 205-223, 2015.

[14] Gunes, B. and Kizilcik, H. S. Developing Three-Tier Misconception Test about Regular Circular Motion, Hacettepe University Journal, 2011.

[15] Erceg, N. et al. Probing students' conceptual knowledge of satellite motion through the use of diagram, Revista Mexicana de fisica E, Vol. 60, 75-85, 2014.

[16] Finley, F. N., Stewart, J., \& Yarroch, W. L. (1982), Teachers' perceptions of important and difficult science content. Science Education, 66 (4), 531-538.

[17] Salvage M. D., \& Williams, J. S. (1989). Centrifugal force: Fact or fiction? Physics Education, 24, 133-140.

[18] Warren, J. W. (1971). Circular Motion. Physics Education, 6, 74-77.

[19] Viennot, L. (1979). Spontaneous reasoning in elementary dynamics. European Journal of Science Education. 1 (2), 205221.

[20] McCloskey, M., Caramazza, A., \& Green, B. (1980). Curvilinear motion in the absence of external forces: Naïve beliefs about the motion of objects. Science, 210 (5), 1139-1141.

[21] McCloskey, M., \& Kohl, D. (1983). Naïve Physics: The curvilinear impetus principle and its role in interactions with moving objects. Journal of Experimental Psychology: Learning, Memory \& Cognition, 9 (1), 146-156.

[22] Gunstone, R. (1984). Circular motion: Some pre-instructional alternative frameworks. Research in Science Education, 14, $125-135$.

[23] Gardner, P. L. Circular motion: Some post-instructional alternative frameworks. Research in Science Education, 1984, 14, 136-145. 
[24] Searle, P. (1985). Circular motion concepts of first year engineering students. Research in Science Education, 15, 140150 .

[25] Whiteley, P. (1995). Students' difficulties with the force(s) acting on the Moon. Physics Education, 30, 31-33.

[26] Chambers, S. K., \& Andre, T. (1997). Gender, Prior Knowledge, Interest, and Experience in Electricity and Conceptual Change Text Manipulations in Learning about Direct Current. Journal of Research in Science Teaching, 34, 107-123. http://dx.doi.org/10.1002/(SICI)10982736(199702)34:2<107: AID-TEA2>3.0.CO;2-X.

[27] Gunstone, R. F. (1995). The Importance of Specific Science Content in the Enhancement of Metacognition. In P. J. Fensham, R. F. Gunstone, \& R. T. White (Eds.), The Content of Science A Constructivism Approach to Its Teaching and Learning (pp. 131-146). London The Falmer Press.

[28] Tsai, C. C. (1999). Overcoming Junior High School Students' Misconceptions about Microscopic Views of Phase Change: A Study of an Analogy Activity. Journal of Science Education and Technology, 83-91. http://dx.doi.org/10.1023/A:1009485722628

[29] Sencar, S., \& Eryilmaz, A. (2004). Factors Mediating the Effect of Gender on Ninth-Grade Turkish Students' Misconcep- tions Concerning Electric Circuits. Journal of Research in Science Teaching, 41, 603-616. http://dx.doi.org/10.1002/tea.20016

[30] Wong, T. K., \& Suleiman, S. (2008). The Level of Alternative Framework among Form Five Science Stream Students on the Topic of Buoyancy. National Conference of Science and Mathematics Education, Johor, Malaysia. http://eprints.utm.my/7669/

[31] Halim, A., Meerah, T., \& Halim, L. (2009). Development and Application of Diagnostic Test to Identify Students' Misconception on Quantum Physics. Sains Malaysiana, 38, 543-551.

[32] Osman, K., Halim, L., \& Meerah, T. S. M. (2006). What Malaysian Science Teachers Need to Improve Their Science In- structions: A Comparison a Cross Gender, School Location and Area of Specialization. Eurasia Journal of Mathematics, Science and Technology Education, 2, 58-81.

[33] Styer, F. D. (1995). Common Misconceptions Regarding Quantum Mechanics. American Journal of Physics, 64, 31-34. http://dx.doi.org/10.1119/1.18288

[34] Abell, S. K. \& Lederman, N. G. (2007). Handbook of Research on Science Education. Mahwah, NJ: Lawrence Erlbaum as associates.

[35] Redish, E. F. (2004). A theoretical framework for physics education research: modelling students thinking ProcVarenna Summer School, 'Enrico Fermi' Course CLVI Varennaed E F Redish and M Vicentini (Amesterdam: ISO).

[36] Planinic, M., boone, W. J., Krsnik, R. and Beilfuss, M. L. (2006). Exploring alternative conceptions from Newtonian dynamics and simple DC circuits: links between item confidence. J. Res. Sci; Teach, 43, 150-71.

[37] Viennot. L. (2002)."Teaching Physics", 'Laws that hurt common sense'. Éditeur: De Boeck Supérieur.

[38] Hestenes, D., Wells, M. and Swachamer, G. (1992) Force concept inventory. The Physics Teacher, 30, 141-153.

[39] Gauld, C. (1998) Solutions to the problem of impact in the $17^{\text {th }}$ and $18^{\text {th }}$ centuries and teaching Newton's third law today. Science and Education, 7, 49-67.

[40] Montanero, M., Suero, M. I., Perez, A. L. and Pardo, P. J. (2002) Implicit theories of $s$ tatic interactions between two bodies. Physics Education, 37, 318-323.

[41] Hellingman, C. (1992). Newton's third law revisited. Physics Education, 127, 112-115.

[42] De Jong, M. L. (1988). What name should be used for the force required to move a mass on a circle? 1. Centripetal force, 2. Centrifugal force, 3. None of the above. The Physics Teacher, 26, 470-1.

[43] Smith, P. A. (1992). Let us get rid of "centripetal force". Phys. Teach, 30, 316-317.

[44] Galili, I. \& Hazan, A. (2000) Learners' knowledge in optics: interpretation, structureand analysis. International Journal of Science education, 22, 57-88.

[45] Yip, D. Y. (1998). Identification of misconceptions in novice biology teachers and remedial strategies for improving biology learning. International journal of Science education, 20, 461-477.

[46] Gopal, H., Kleinsmidt, J. \& Case, J. (2004). An investigation oftertiarystudents' understanding of evaporation, condensation and vapor pressure. International of science education, 26, 1597-1620.

[47] Minner, D. D., Levy, A. J., and Century, J. Inquiry-based science instruction-what is it and does it matter? Results from a research synthesis year 1984-2002, Journal of Research in Science Teaching, Vol. 47, No. 4, 474-496, 2010.

[48] Handelsman, J., Ebert-May, D., Beichner, R., Bruns, P. et al. Scientific Teaching, Science, New Series, Vol. 304, No. 5670, 521-522, 2004.

[49] von Secker, C. Effects of Inquiry-Based Teacher Practices on Science Excellence and Equity. The Journal of Educational Research, Vol. 95, No. 3, 151-160, 2002.

[50] Darling-Hammond, L. Teacher Quality and Student Achievement. Education Policy Analysis Archive, Vol. 8, No. 1,2000

[51] Goldhaber, D. D. Does Teacher Certification Matter? High School Teacher Certification Status and Student Achievement. Educational Evaluation and Policy Analysis, Vol. 22. No. 2, 129-145, 2000. 